\title{
Dynamic changes in uterine NK cell subset frequency and function over the menstrual cycle and pregnancy
}

\section{Emily M Whettlock ${ }^{1 \dagger}$, Ee Von Woon ${ }^{1 \dagger}$, Antonia O Cuff ${ }^{1}$, Brendan Browne ${ }^{1}$, Mark R Johnson ${ }^{1}$, Victoria Male ${ }^{1}$}

$5 \quad{ }^{1}$ Department of Metabolism, Digestion and Reproduction, Institute of Developmental Reproductive

6 and Developmental Biology, Imperial College London, Chelsea and Westminster Hospital Campus,

7369 Fulham Road, London, SW10 9NH, United Kingdom

$8 \uparrow$ These authors have contributed equally to this work and share first authorship.

9 * Correspondence:

10 Victoria Male

11 v.male@imperial.ac.uk

12 Keywords: NK cells, ILC, decidua, endometrium, pregnancy, single cell analysis

\section{Abstract}

14 Uterine Natural Killer cells ( $\mathrm{uNK}$ ) play an important role in promoting successful pregnancy by regulating trophoblast invasion and spiral artery remodelling in the first trimester. Recently, single cell RNA sequencing (scRNAseq) on first trimester decidua showed that uNKs can be divided into three subsets, which may have different roles in pregnancy. Here we present an integration of previously published scRNAseq datasets, together with novel flow cytometry data to interrogate the frequency, phenotype and function of uNK1-3 in seven stages of the reproductive cycle (menstrual, proliferative, secretory phases; first, second, third trimester; and postpartum). We found that uNK1 and -2 peak in the first trimester, but by the third trimester the majority of uNK are uNK3. All three subsets are most able to degranulate and produce cytokines during the secretory phase of the menstrual cycle and express KIR2D molecules, which allow them to interact with HLA-C expressed by placental extravillous trophoblast cells, at the highest frequency during the first trimester. Taken together, our findings suggest that uNK are particularly active and able to interact with placental cells at the time of implantation, and that $\mathrm{uNK} 1$ and 2 may be particularly involved in these processes. Our findings are the first to establish how uNK frequency and function changes dynamically across the healthy reproductive cycle. This serves as a platform from which the relationship between uNK function and impaired implantation and placentation can be investigated. This will have important implications for the study of subfertility, recurrent miscarriage, pre-eclampsia and pre-term labour. 


\section{Introduction}

Uterine Natural Killer cells (uNK) are NK-like cells that are found in the lining of the uterus (called "decidua" in pregnancy and "endometrium" outside of pregnancy). They are distinct from peripheral blood NK (pNK) in both phenotype and function. Unlike pNK, uNK are only weakly cytotoxic and instead produce factors that are pro-angiogenic and that attract fetally-derived placental cells called extravillous trophoblast (EVT) (1-6). uNK are most prominent in the first trimester of pregnancy, at which time they account for 70-80\% of immune cells in the decidua (7). Their prominence at the time of implantation, and production of factors that are predicted to promote trophoblast invasion and spiral artery remodeling, indicates they are likely to have a role in placental implantation.

Further evidence for a role of $\mathrm{uNK}$ in implantation comes from their expression of high levels of

killer-cell immunoglobulin-like receptors (KIRs), CD94/NKG2 (8, 9), and LILRB1 (9), which allows them to recognize the human leukocyte antigens (HLAs) expressed by EVT: HLA-C, HLA-E and HLA-G respectively (10-12). Immunogenetic studies demonstrate that combinations of HLA and pre-eclampsia, fetal growth restriction and recurrent miscarriage, suggesting that uNK activation via KIR is important for implantation (13-20). The increased expression of KIRs by uNK around the time of implantation provides additional support for this idea (21).

Until recently, it was thought that uNK formed a single population, but single cell RNA sequencing (scRNAseq) has now demonstrated three subpopulations of uNKs in first trimester decidua (22). non-pregnant endometrium (23). Here, we call these subsets "uNK1", "uNK2", and "uNK3" in recognition of the finding that they are not confined to the decidua. uNK1 express higher levels of uNK3 produced more cytokines upon stimulation, indicating their role may be immune defense (24). However, several questions remain open. Are these three subpopulations still present at the end of pregnancy? Do the subpopulations change in prominence and/or activity over the reproductive cycle? The answers to these questions could elucidate which subpopulations are important in implantation, parturition, and immune protection throughout the reproductive cycle.

Here, we show that the proportions of the uNK subpopulations remain stable through the menstrual uNK1 are more prominent in the first trimester of pregnancy, potentially indicating a requirement for this subset in the mediation of implantation whereas uNK3 are the most prominent at the end of pregnancy. Overall, we outline how the three uNK subpopulations change in proportion phenotype and function throughout the reproductive cycle.

\section{Materials and Methods}

70 Primary tissue

71 Collection of human tissue was approved by London - Chelsea Research Ethics Committee (study

72 numbers: 10/H0801/45 and 11/LO/0971). 
73

29 endometrial samples were taken by Pipelle biopsy before insertion of intrauterine device for contraception. Samples taken on a day of bleeding were assigned as menstrual phase. Other samples were categorized to proliferative or secretory phase by date of last menstrual period and serum progesterone level. Samples obtained before Day 14 were assigned as proliferative phase and after day 14 as secretory phase. This was confirmed retrospectively by serum progesterone level, according to previously published reference range (25). Samples from postpartum participants were assigned by number of days from delivery of the baby up until 16 weeks post-partum.

10 decidual samples were taken from participants undergoing surgical management of elective termination of pregnancy between 6 to 13 weeks of pregnancy and 10 from participants undergoing elective caesarean sections, over 37 weeks of pregnancy and not in labour. For labouring data, a following 8 samples were taken from participants in the early stages of labour $(1-3 \mathrm{~cm}$ cervical dilation and regular contractions) who had a caesarean section, and 5 samples were taken from participants after a vaginal birth. Matched peripheral venous blood was obtained from all patients at time of obtaining endometrial or decidual samples. Patient characteristics are summarized in Suppl tables 1 and 2 .

Lymphocytes were extracted from peripheral blood by layering onto Histopaque (Sigma Aldrich), spinning down $\left(700 \mathrm{xg}, 20\right.$ minutes, $\left.21^{\circ} \mathrm{C}\right)$ and retrieving the interface which was washed twice with Dulbecco's Phosphate-Buffered Saline (Life Tech) (500 xg, 10 minutes, $\left.4^{\circ} \mathrm{C}\right)$. Briefly, endometrial tissue was passed through $100 \mu \mathrm{m}$ cell strainer, pelleted $\left(700 \mathrm{xg}, 10\right.$ minutes, $\left.4^{\circ} \mathrm{C}\right)$, resuspended in Dulbecco's Phosphate-Buffered Saline supplemented by 10\% Fetal Calf Serum (Sigma Aldrich), passed through $70 \mu \mathrm{m}$ strainer, and layered on Histopaque as above.

For first trimester samples, decidua compacta was extracted from products of conception and stirred for 20 minutes to remove blood before mincing with scalpel followed by GentleMACS dissociation (Miltenyi). Minced tissue was passed through $75 \mu \mathrm{m}$ sieve, pelleted $\left(500 x g, 10\right.$ minutes, $\left.4^{\circ} \mathrm{C}\right)$ and resuspended in PBS/1\% FCS before passing through $100 \mu \mathrm{m}$ strainer. Filtrate was layered on Histopaque as above.

For third trimester decidua basalis (DB) samples, small sections were cut from the maternal side of the placenta and washed using a magnetic stirrer in $\mathrm{Mg}^{2+}$ and $\mathrm{Ca}^{2+}$ free PBS (Gibco) for 20 minutes. Blood clots, vessels and placental tissue were physically removed and cleaned decidual tissue was placed in new $\mathrm{Mg}^{2+}$ and $\mathrm{Ca}^{2+}$ free PBS. The tissue was spun (400 xg, 5 mins $21^{\circ} \mathrm{C}$ ) and PBS removed. The tissue was resuspended in Accutase (Invitrogen), mechanically digested in $\mathrm{C}$ tubes using a GentleMACs dissociater and placed in a $37^{\circ} \mathrm{C}$ shaking water bath for 45 minutes. Minced tissue was passed through a $70 \mu \mathrm{m}$ strainer, resuspended in PBS/1\%FCS/2mM EDTA. Filtrate was layered on Histopaque as above. For the third trimester decidua parietalis (DP) samples, $10 \mathrm{~cm} \mathrm{x}$ $10 \mathrm{~cm}$ sections of the fetal membrane were dissected and the decidua removed using a cell scraper (Starsted). The tissue underwent enzymatic and mechanical digestion as described in the decidua basalis protocol. 
116 Extracted lymphocytes were counted by light microscopy (Leica) with a haemocytometer. A total of

$1170.2 \times 10^{6}$ to $1 \times 10^{6}$ cells per condition were allocated for phenotype and functional assessment.

\section{Stimulation with PMA/ionomycin}

21 endometrial, all first trimester and all third trimester samples were used for functional assessment.

Endometrial lymphocytes were stimulated immediately after isolation, and decidual lymphocytes were stimulated after 12 to 20 hours of rest at $37^{\circ} \mathrm{C}$. Optimization experiments showed no difference between cells stimulated fresh and after rest (Supp Fig 1).

For functional assessment, cells were suspended in RPMI enriched with antibiotics, EDTA and sodium pyruvate and divided into unstimulated and stimulated wells. Anti-CD107a BV605 (100 $\mu \mathrm{l} / \mathrm{ml})$, Brefeldin $(10 \mu \mathrm{g} / \mathrm{ml})$ and Monensin $(2 \mu \mathrm{M} / \mathrm{ml})$ were added to all wells and Phorbol 12myristate 13 -acetate (PMA) $(50 \mathrm{ng} / \mathrm{ml})$ and ionomycin $(1 \mu \mathrm{g} / \mathrm{ml})$ into the stimulated wells only. Cells were incubated for 4 hours at $37^{\circ} \mathrm{C}$ then stained with antibodies. For third trimester samples, cells were incubated for 6 hours with anti-CD107a, PMA and ionomycin, with Brefeldin and Monensin added 2 hours into the incubation.

134 For the scRNAseq data, R(26) was used for the majority of the analysis. This included use of the

A scRNA seq dataset from the non-pregnant uterus (available at www.reproductivecellatlas.org/) was converted from a Python format into a R Seurat object. The object was subset to cells that had been classified as "Lymphoid" or "Myeloid" under "Cell.type", in the metadata.

A scRNAseq dataset from the first trimester uterus (available at Array Express E-MTAB-6701) was converted from .txt into a R seurat object. The object was subset to cells that had been designated an immune cell type, e.g. "dNK1" under "Annotation", in the metadata. Cells originating from placenta or blood were removed, so only decidual cells remained. Data from one donor was removed from the analysis due to their NK cells clustering independently of all other NK cells.

The scRNAseq dataset (dbGaP phs001886.v1.p1, reanalyzed with permission of the NIH, project ID 26528) contained samples from one second trimester accreta sample and 9 third trimester participants. Both datasets were filtered, aligned and quantified using Cell Ranger software (version 5.0.1, 10x Genomics). h19 was used as a human genome reference. Downstream analyses were performed using the R package Seurat(27). Cells with fewer than 200 genes and genes that were expressed in less than 10 cells were removed. Furthermore, cells where the gene content was greater than 10\% mitochondrial genes were removed. Clusters were identified using "FindClusters" algorithm. The "FindAllMarkers" algorithm was used to identify the immune clusters, and subset the 
included in the analysis looking at $\mathrm{dNK}$ across the reproductive cycle. PTL samples were not included in the term labouring analysis.

The four datasets were integrated based on a previously published workflow (36). Clusters that appeared to be non-immune cells were removed and the remaining cells were reanalysed using the same workflow. The algorithm "FindConservedMarkers" was used to identify the clusters dNK1, $\mathrm{dNK} 2$ and dNK3. This was confirmed by the metadata column "annotation" from the first trimester dataset. For visualisation of clusters and gene expression across the reproductive cycle, each dataset was downsampled so the total number of cells displayed was equal in each dataset.

\section{Flow cytometry}

The following anti-human antibodies were used: Anti-CD56 Brilliant Violet (BV) 650 (clone NCAM 16.2, BD Bioscience), anti-CD39 BV421 (clone A1, Biolegend), anti-CD3 BV711 (clone SK7, Biolegend), anti-CD103 BV785 (clone Ber-ACT8, Biolegend), anti-CD16 Alexa Fluor(AF)700 (clone 3G8, Biolegend), anti-CD9 phycoerythrin(PE)/Dazzle 594 (clone HI9a, Biolegend), antiCD49a PE/Cy7 (clone TS2/7, Biolegend), anti-CD45 allophycocyanin (APC) (clone HI30, Biolegend), anti-CD94 PE (Clone HP-3D9, BD Bioscience), anti-CD158a/h (KIR2DL1/DS1) VioBright 515 (clone REA1010, Miltenyi Biotec), anti-CD158b (KIR2DL2/DL3) APC vio 770 (clone REA 1006, Miltenyi Biotec), CD85j (ILT2 or CD94) Peridinin chlorophyll protein (PerCP)eFluor 710 (clone HP-F1, Thermo Fisher Scientific) and anti-CD107a BV605 (clone H4A3, Biolegend) for surface antigens, and anti-IL-8 PE (clone G265-8, BD Bioscience), anti-IFN- $Y$ APCvio770 (clone REA600, Miltenyi Biotec), anti GM-CSF PERCP/Cyanine 5.5 (clone BVD221C11, Biolegend), anti-TNF $\alpha$ FITC (clone MAb11, Biolegend) for intracellular staining. Cells were first incubated with fixable viability dye (Live/Dead Fixable Aqua Dead Cell stain kit, LifeTech) $\left(15\right.$ minutes, $\left.4^{\circ} \mathrm{C}\right)$ followed by incubation with surface antibodies $\left(15\right.$ minutes, $\left.4^{\circ} \mathrm{C}\right)$. For intracellular staining, human FoxP3 buffer (BD Bioscience) was used according to manufacturer's instructions before staining with intracellular antibodies $\left(30\right.$ minutes, $\left.4^{\circ} \mathrm{C}\right)$. For third trimester samples, fixable viability dye was included with the surface staining antibodies (20 minutes, RT) and intracellular staining used the Cytofix/Cytoperm kit (BD Biosciences) according to manufacturer's instructions. Excess antibodies were washed off ( 5 minutes, $\left.500 \mathrm{xg}, 4^{\circ} \mathrm{C}\right)$ between each incubation and twice after final incubation with intracellular antibodies.

\section{Statistical analysis}

Data were acquired on an BD Fortessa and analysed using FlowJo (Tree Star, Ashalnd, OR). Application settings were used to ensure reproducible results. Statistical analysis were performed using PRISM (GraphPad Software Inc.). Data were assessed for normality using Shapiro-Wilk tests to determine whether a parametric or a non-parametric statistical test was appropriate. The appropriate statistical test was used to compare subsets as specified in figure legends. $\mathrm{p}<0.05$ was considered significant.

\section{Results}


We examined tissues and collected data at seven stages of the reproductive cycle. In the menstrual cycle there are three stages: menstrual (when the lining of the uterus is shed), proliferative (prior to ovulation) and secretory (after ovulation). Pregnancy is divided into three trimesters: first (1-12 weeks), second (12-28 weeks), third ( $28-40$ weeks). We also examined postpartum samples (up to 16 weeks post-delivery). For the scRNAseq data, 5 stages were examined: proliferative, secretory, first trimester, second trimester and third trimester. For flow cytometry, 6 stages were examined: menstrual, proliferative, secretory, first trimester, third trimester and postpartum.

During the menstrual cycle stages the uterine tissue we examined is known as the endometrium. During pregnancy, this tissue undergoes a process called decidualization and results in three tissues known as the decidua basalis (which lines the maternal side of the placenta), decidua parietalis (which lines the rest of the uterus) and decidua capsularis (which lines the embryo on the luminal side). When taking samples from first trimester tissue, it not possible to differentiate between the different decidual tissues. During second trimester the decidua capsularis fuses with the decidua parietalis. When taking samples from third trimester tissue, it is possible to get distinct samples from the decidua basalis and the decidua parietalis.

uNK1, -2 and -3 are present throughout the human reproductive cycle and vary in frequency scRNAseq analysis has previously identified that three subpopulations of uNK, uNK1, -2 and -3 present in first trimester decidua (22) and non-pregnant endometrium (23). Previous analysis of scRNAseq data from third trimester decidua identified only a single cluster within the uNK population (37), and our reanalysis of the third trimester dataset alone confirmed this. However, when the third trimester data was integrated with data from the non-pregnant uterus, first and second trimester, the third trimester dNK cells did form three clusters (Fig. 1a, b).

In the first trimester, uNK can be distinguished from circulating NK cells by their expression of CD49a and CD9; the subsets are then defined by their expression of CD39 and CD103 (22). We confirmed the presence of CD49a+ uNK in endometrium and in first and third trimester decidua, and that the three subpopulations uNK1, -2 and -3 can be identified using CD39 and CD103 (Fig 2a). However, in third trimester samples there was a significant CD49a+CD9- population. A comparison of CD49a+CD9+ and CD49a+ CD9- detected no phenotypic differences between these two populations, suggesting that CD49a alone can be used to identify uNK cells in the third trimester (Supp Fig 2). For consistency of gating strategy, we also identified uNK cells by their expression of CD49a alone in endometrial, first trimester and postpartum samples.

In line with previous reports (7), we observed a peak in total uNK, as a proportion of total CD45+ lymphocytes, in first trimester pregnancy by both scRNAseq and flow cytometry (Figure 1c, Fig 2b). We observed a similar proportion of total $\mathrm{UNK}$ in proliferative and secretory phase (Fig. 1c, 2c), in contrast to a previous report of higher proportion of uNK in secretory phase (38). This may be due to our use of CD49a allowing the removal of contaminating pNK from analysis, or due to their more vigorous sub-classification of the secretory stage. 
Next, we examined uNK 1, -2 and -3 frequency expressed as either a proportion of total CD45+ lymphocytes or total uNK. We observed an increase in frequency of uNK1 when transitioning from secretory phase to first trimester pregnancy, but this was not sustained into third trimester decidua. This observation applied to both expression of uNK1 as percentage of both CD45+ lymphocytes and percentage of total NK cells. (Fig. 1c and Fig 2b), and the change was significant when measured by flow cytometry.

The variation of uNK2 frequency was similar to that observed for $\mathrm{uNK} 1$, with a peak in the first trimester observed by scRNAseq, and flow cytometry when frequency was measured as a percentage of CD45+ lymphocytes (Fig 1c and 2b). For the latter, uNK2 were significantly higher in the first trimester, compared to the third. Further, there was an upward trend of uNK2 when transitioning from third trimester decidua to postpartum endometrium when measured as a proportion of total NK (Fig 2c).

253

For uNK3, there was no change in frequency through the menstrual cycle. When measured as a percentage of CD45+ lymphocytes, there was a reduction in uNK3 in third trimester decidua parietalis, compared to both first trimester decidua and third trimester decidua basalis. This was there was a dip in the first trimester and a peak in both types of third trimester decidua. This was significant when measured by flow cytometry. The discrepancy between the proportions when expressed as a percentage of CD45+ lymphocytes or total uNK cells is likely due to the change in frequency of total $\mathrm{uNK}$, as a proportion of CD45+ lymphocytes.

Within the third trimester decidua, the uNK2 population appeared greater in proportion of total uNK in the decidua parietalis compared to the decidua basalis in both scRNAseq and flow cytometry, although this did not reach significance for either (Fig 1c and $2 b$ ). The uNK3 population appeared greater in the decidua basalis, compared to decidua parietalis, which was significant when measured by flow cytometry as a percentage of CD45+ lymphocytes (Fig. 1c and 2b).

Peripheral blood NK cell frequency does not vary over the reproductive cycle or correlate with uNK frequency

We also examined CD56Bright and Dim NK cells in matched peripheral blood by a conventional gating strategy to identify these populations. (Fig 2c). Unlike uNK, there was no variation in total CD56+pNK, CD56Bright or CD56Dim in peripheral blood when transitioning through different phases of the reproductive cycle. Furthermore, there was no significant correlation in levels of $\mathrm{pNK}$ and uNK subsets when expressed either as proportion of CD45+ live lymphocytes or total NK cells. (Supp Fig 3).

uNK subsets upregulate KIR and LILRBI during transition from non-pregnant endometrium to first trimester decidua

280 We next examined uNK expression of receptors that interact with trophoblast cells: KIR2DL1 and

281 KIR2DL2/3 recognise HLA-C, LILRB1 recognises HLA-G and CD94 recognises HLA-E (39). 
In line with earlier findings on first trimester $\mathrm{uNK}(22,24)$, we observed that uNK1 expressed higher levels of KIR than uNK2 and -3 (Fig 3b,c). We also found that all three uNK subsets expressed increased KIR in the first trimester of pregnancy, compared to non-pregnant endometrium and third trimester decidua (Fig 3b,c). Similar to KIR, LILRB1 protein expression peaked in the first trimester, although this was only statistically significant in uNK2 and uNK3 (Fig 3b). LILRB1 transcript expression followed a similar trend, although in contrast to our findings at the protein level, LILRBI mRNA was not detectable in the decidua basalis (Fig 3c). At the transcript and protein level, CD94 was expressed at a higher level on uNK2 and 3 compared to uNK1 (Fig.3b, c). There was a slight reduction in CD94 transcript (KLRD1) towards the end of pregnancy, but this was not observed at the protein level. (Fig. 3b, c)

In line with our finding that pNK did not change in frequency over the reproductive cycle, examination of NK cells from matched blood showed no change in the frequency at which KIR, LILRB1, and CD94 are expressed in these cells. (Fig. 3b).

\section{$u N K$ are the most active at the time of implantation} We next assessed functional responses with and without stimulation with PMA and ionomycin (Fig. 4a). CD107a staining is a proxy for degranulation and previous studies have shown that this acts as a reliable measure of overall uNK activation (40). We also examined the production of IL-8 and GMCSF, which are thought to promote EVT invasion $(6,40,41)$, and the classical NK cell cytokines IFN $\gamma$ and TNF $\alpha$ (Fig. 4b).

Degranulation in unstimulated conditions declined during the proliferative phase, slightly in uNK2 and significantly in uNK3, compared the other two phases of the menstrual cycle. (Fig. 4b). At the end of pregnancy, degranulation was significantly lower in third trimester decidua parietalis compared to decidua basalis in $\mathrm{dNK} 1$, but this was not replicated in the other subsets. (Fig. 4b). In stimulated cells there was a reduction in degranulation in $\mathrm{uNK} 2$ and $\mathrm{uNK} 3$ in both third trimester decidua compared to first trimester decidua (Fig. 4b).

For TNF- $\alpha$, IFN- $\gamma$ and IL-8, we observed peaks in cytokine production across all stimulated uNK subsets during secretory phase, compared to the proliferative phase and first trimester, although this did not reach statistical significance in all cases (Fig 4b). For IL-8 in uNK3 this peak was maintained into first trimester pregnancy. This trend was also present in unstimulated cells for IL-8 (Fig 4b).

Third trimester uNK produced less cytokine than first trimester uNK, although this only reached significance in IL-8 production from $\mathrm{uNK} 3$. This reduction in cytokine production through pregnancy was also seen at the mRNA level for IL-8 (Fig. 4c). In contrast, GM-CSF protein production was consistently low in the menstrual cycle, including the secretory phase, but increased significantly in the first trimester of pregnancy in unstimulated uNK1 and stimulated uNK3. (Fig. 
323 For examination of NK cells from matched peripheral blood, data from CD56Brights and Dims are shown together due to downregulation of CD16 after stimulation. Aside from a significant decline in CD107a expression in unstimulated cells when transitioning from secretory phase to first trimester pregnancy, there were no distinct trend in both stimulated and unstimulated cells (Fig. 4b).

The mRNA expression of other NK cell proteins of interest across the reproductive cycle, such as granzymes, that were not included in the flow cytometry panel, can be seen in the supplementary in labouring compared to non-labouring decidua (Fig 5b). The proportion of uNK in non-labouring decidua was lower in the decidua parietalis compared to the decidua basalis (Fig 5b). This is in contrast to a previous report which found a higher frequency of CD56 bright NK cells in the parietalis than the basalis (44). However, the lack of tissue-specific markers means that it is difficult to be sure if these all represent $\mathrm{uNK}$.

We next examined the uNK subpopulations in non-labour compared to early labour and established labour samples. We observed no change in the frequency of any of the uNK or pNK subsets across the spectrum of these samples (Fig. 5a and 5b). The receptors examined were also stable during labour (Supp Fig 6), an observation that suggests that EVT cross-talk with uNK is not a major participant in labour. Similarly, for those markers examined, the function of uNK and pNK subsets remain stable during labour, although we did observe that, regardless of labouring state, the uNK1 population in the decidua basalis is significantly more active than the population in the decidua parietalis (Figure 5 and supp fig 7). This is supported by the low number of differentially expressed genes across all three uNK subsets in the scRNAseq data comparing non-labour to labour samples (Supp table 3).

\section{Discussion}

To our knowledge, this is the first study to track the three uNK subpopulations throughout the reproductive cycle, looking at their frequency, phenotypes and functions. In line with previous studies, we found that the total uNK population peaked during the first trimester of pregnancy (7, 45). We discovered that uNK1 and uNK2 peak in this period, but that uNK3 peaks towards the end of pregnancy. This aligns with a recent report that KIR+CD39+ uNK (mostly representing uNK1) and KIR+CD39- (mostly representing uNK2) increase in frequency towards the end of the menstrual cycle and remain elevated in early pregnancy (46). Both these reports support the proposal that uNK1 communicate with EVTs in early pregnancy $(22,24)$, but may also point to a role for uNK2 in this process.

It has previously been reported that KIR expression by total uNK increases in the first trimester, compared to non-pregnant endometrium (21). Our finding that the major KIR-expressing subset, $\mathrm{uNK} 1$, is most frequent in the first trimester might suggest that this increase in KIR expression is at 
increased KIR in the first trimester of pregnancy. A similar trend was seen for LILRB1. This suggests that all uNK subpopulations increase their ability to recognise EVT, via HLA-C and HLA$\mathrm{G}$, in the first trimester. In comparison, CD94 expression was higher on uNK2 and -3 subsets compared to uNK1. This is in contrast with previous findings (24). However, this measured marker intensity on recovered cryopreserved cells, whereas we report percentage of CD94+ fresh cells. Therefore, it is possible that the freezing process preferentially killed CD94- uNK1 cells or uNK1 have a lower percentage of cells expressing CD94, but have a higher expression per cell. typically most active at around the time of implantation, in the secretory phase of the menstrual cycle and the first trimester of pregnancy. This is in line with previous findings that first trimester uNK are more able to degranulate in response to HCMV-infected targets than those at term, although the same study found that, following IL-15 stimulation, term uNK are better able to degranulate in response to PMA and ionomycin than first trimester cells (9). Without stimulation, uNK produce little IFN $\gamma$, IL8, TNF $\alpha$ and GM-CSF. However, after stimulation with PMA and ionomycin, uNK have the highest ability to produce most of these cytokines during the secretory phase, except for GM-CSF, whose production peaks in the first trimester. This finding is interesting because the timing of maximum activation coincides with the window of implantation, suggesting that $\mathrm{uNK}$ may have a role in coordinating successful implantation.

Cumulative evidence indicates an important physiological role for uNK in first trimester pregnancy, but there is conflicting evidence on their role in reproductive failure. Our findings and those of others $(22,24)$ point to uNK1 as the uNK subset more likely to mediate placental implantation in early pregnancy. Future studies focusing specifically on this subset may be able to elucidate differences that were previously masked due to examination of $\mathrm{uNK}$ as a bulk population. A recent study using scRNAseq suggests that there is a reduction of uNK1 in pathological pregnancies (47); however, these findings need to be interpreted with caution because the pathological samples were collected after pregnancy loss, making it difficult to discern if changes seen in immune cells are a cause or an effect, due to inflammatory changes that typically occur after fetal demise. To overcome this, future studies could interrogate uNK during window of implantation (48) or from elective termination of pregnancy samples stratified to low and high risk by uterine artery doppler which has high specificity in predicting risk of pre-eclampsia and intrauterine growth restriction (49).

In the third trimester the majority of uNK cells are uNK3, which express low levels of KIRs and LILRB1. This could suggest that, in contrast to early pregnancy, the major role for uNK in late pregnancy does not involve interactions with EVTs. Similarly, the expression of the functional markers we examined was lower in the third trimester. This could indicate that, if these cells have a role at the end of pregnancy, it is via a different mechanism of action. Intriguingly, by scRNAseq, uNK3 were the most transcriptionally different between non-labouring and labouring states, suggesting they may have a role in labour that is yet to be defined. It would be interesting to do a broader analysis of these cells, or examine how they look in pathological cases such as pre-eclampsia or preterm birth. 
408 In conclusion, we show here how uNK subset number, expression of receptors and function change 409 dynamically across the healthy reproductive cycle. This provides evidence on their physiological role 410 in implantation, but will also provide an important platform from which the relationship between $411 \mathrm{uNK}$ function and pathologies of pregnancy associated with impaired implantation and placentation 412 can be investigated. 


\section{Availability of data and code}

414 For scRNAseq analyses, data is available from: http://www.reproductivecellatlas.org/ (non-pregnant

415 uterus), Array Express E-MTAB-6701 (first trimester), dbGaP phs001886.v1.p1 (second and third

416 trimester). Code is available from https://github.com/ewhettlock/reproductive_cycle. Flow cytometry

417 data is available from https://osf.io/wkxyz/.

418

419 Conflict of Interest

420 The authors declare that the research was conducted in the absence of any commercial or financial

421 relationships that could be construed as a potential conflict of interest.

422

423

\section{Author Contributions}

424 EMW, EVW and VM designed the study, analysed results and wrote the manuscript. EMW, EVW and AOC carried out experiments. EVW, BB and MRJ consented patients and collected clinical samples. All others contributed to editing the manuscript.

\section{Funding}

429 This study was funded by Borne.

\section{Acknowledgements}

432 We would like to thank all the people from Chelsea and Westminster Hospital,West Middlesex

433 University Hospital and John Hunter Clinic (London, UK) who contributed samples to this study. We

434 would also like to thank Dr Pei Lai, Dr Nishel Shah, Miss Sharmista Guha and all the clinical staff

435 who helped in the collection of samples. 


\section{References} phenotype and cytotoxic activity of lymphocytes from human term decidua against trophoblast. $\mathbf{J}$ Reprod Immunol. 1996;31(1-2):109-23. K562 cell line but not against first trimester trophoblast. Cell Immunol. 1989;118(2):337-44. 3. Koopman LA, Kopcow HD, Rybalov B, Boyson JE, Orange JS, Schatz F, et al. Human decidual natural killer cells are a unique NK cell subset with immunomodulatory potential. J Exp Med. 2003;198(8):1201-12.

450 5. Siewiera J, El Costa H, Tabiasco J, Berrebi A, Cartron G, Le Bouteiller P, et al. Human cytomegalovirus infection elicits new decidual natural killer cell effector functions. PLoS Pathog. 2013;9(4):e1003257.

453 6. Hanna J, Goldman-Wohl D, Hamani Y, Avraham I, Greenfield C, Natanson-Yaron S, et al. Decidual NK cells regulate key developmental processes at the human fetal-maternal interface. Nat Med. 2006;12(9):1065-74.

456 7. Williams PJ, Searle RF, Robson SC, Innes BA, Bulmer JN. Decidual leucocyte populations in early to late gestation normal human pregnancy. J Reprod Immunol. 2009;82(1):24-31.

8. $\quad$ King A, Hiby SE, Verma S, Burrows T, Gardner L, Loke YW. Uterine NK cells and trophoblast HLA class I molecules. Am J Reprod Immunol. 1997;37(6):459-62. Human Term Pregnancy Decidual NK Cells Generate Distinct Cytotoxic Responses. J Immunol. 2020;204(12):3149-59.

10. King A, Boocock C, Sharkey AM, Gardner L, Beretta A, Siccardi AG, et al. Evidence for the expression of HLAA-C class I mRNA and protein by human first trimester trophoblast. J Immunol. 1996;156(6):2068-76.

11. King A, Allan DS, Bowen M, Powis SJ, Joseph S, Verma S, et al. HLA-E is expressed on trophoblast and interacts with CD94/NKG2 receptors on decidual NK cells. Eur J Immunol. 2000;30(6):1623-31.

12. Kovats S, Main EK, Librach C, Stubblebine M, Fisher SJ, DeMars R. A class I antigen, HLAG, expressed in human trophoblasts. Science. 1990;248(4952):220-3.

471

472 cell immunoglobulin-like receptors and parental HLA-C genotypes with recurrent miscarriage. Hum Reprod. 2008;23(4):972-6.

14. Johnsen GM, Størvold GL, Drabbels JJM, Haasnoot GW, Eikmans M, Spruyt-Gerritse MJ, et al. The combination of maternal KIR-B and fetal HLA-C2 is associated with decidua basalis acute atherosis in pregnancies with preeclampsia. J Reprod Immunol. 2018;129:23-9.

15. Moffett A, Chazara O, Colucci F, Johnson MH. Variation of maternal KIR and fetal HLA-C genes in reproductive failure: too early for clinical intervention. Reprod Biomed Online. 2016;33(6):763-9.

480 16. Cartwright JE, Fraser R, Leslie K, Wallace AE, James JL. Remodelling at the maternal-fetal interface: relevance to human pregnancy disorders. Reproduction. 2010;140(6):803-13.

17. Hiby SE, Walker JJ, O'shaughnessy KM, Redman CW, Carrington M, Trowsdale J, et al. Combinations of maternal KIR and fetal HLA-C genes influence the risk of preeclampsia and reproductive success. J Exp Med. 2004;200(8):957-65. 
18. Hiby SE, Apps R, Sharkey AM, Farrell LE, Gardner L, Mulder A, et al. Maternal activating

KIRs protect against human reproductive failure mediated by fetal HLA-C2. J Clin Invest. 2010;120(11):4102-10. KIR2DS4 Is Expressed by Uterine NK Cells and Contributes to Successful Pregnancy. J Immunol. 2016;197(11):4292-300.

20. Nakimuli A, Chazara O, Hiby SE, Farrell L, Tukwasibwe S, Jayaraman J, et al. A KIR B centromeric region present in Africans but not Europeans protects pregnant women from preeclampsia. Proc Natl Acad Sci U S A. 2015;112(3):845-50.

21. Male V, Sharkey A, Masters L, Kennedy PR, Farrell LE, Moffett A. The effect of pregnancy on the uterine NK cell KIR repertoire. Eur J Immunol. 2011;41(10):3017-27.

22. Vento-Tormo R, Efremova M, Botting RA, Turco MY, Vento-Tormo M, Meyer KB, et al. Single-cell reconstruction of the early maternal-fetal interface in humans. Nature. 2018;563(7731):347-53. 23. Garcia-Alonso L, Handfield L-F, Roberts K, Nikolakopoulou K, Fernando RC, Gardner L, et al. Mapping the temporal and spatial dynamics of the human endometrium $\langle\mathrm{em}>$ in vivo $</ \mathrm{em}>$ and <em>in vitro</em>. Preprint BioRxiv. 2021:2021.01.02.425073. phenotypes and functions of innate lymphoid cells in human decidua during early pregnancy. Nat Commun. 2020;11(1):381.

25. Stricker R, Eberhart R, Chevailler MC, Quinn FA, Bischof P. Establishment of detailed reference values for luteinizing hormone, follicle stimulating hormone, estradiol, and progesterone during different phases of the menstrual cycle on the Abbott ARCHITECT analyzer. Clin Chem Lab Med. 2006;44(7):883-7.

26. Team RC. R: A Language and Environment for Statistical Computing. 2020.

27. Hao Y, Hao S, Andersen-Nissen E, Mauck WM, Zheng S, Butler A, et al. Integrated analysis of multimodal single-cell data. Cell. 2021;184(13):3573-87.e29.

512 28. Wickham H. ggplot2: Elegant Graphics for Data Analysis.: Springer-Verlag New York; 2016.

513 29. Chan C-h, Chan GC, Leeper TJ, Becker J. rio: A Swiss-army knife for data file I/O. 2021.

514 30. Fischer B, Smith M, Pau G. rhdf5: R Interface to HDF5. 2020.

31. Hadley W, Romain F, Lionel H, Kirill M. dplyr: A Grammar of Data Manipulation. 2021.

516 32. Pedersen TL. patchwork: The Composer of Plots. 2020.

517 33. Schauberger P, Walker A. openxlsx: Read, Write and Edit xlsx Files. 2020.

518 34. Ushey K, Allaire J, Tang Y. reticulate: Interface to 'Python'. 2021.

519 35. Wickham H. tidyr: Tidy Messy Data. 2021.

36. Stuart T, Butler A, Hoffman P, Hafemeister C, Papalexi E, Mauck WM, et al. Comprehensive Integration of Single-Cell Data. Cell. 2019;177(7):1888-902.e21.

522 37. Pique-Regi R, Romero R, Tarca AL, Sendler ED, Xu Y, Garcia-Flores V, et al. Single cell transcriptional signatures of the human placenta in term and preterm parturition. Elife. 2019;8.

38. Flynn L, Byrne B, Carton J, Kelehan P, O'Herlihy C, O'Farrelly C. Menstrual cycle dependent fluctuations in NK and T-lymphocyte subsets from non-pregnant human endometrium. Am J Reprod Immunol. 2000;43(4):209-17.

39. Parham P. NK cells and trophoblasts: partners in pregnancy. J Exp Med. 2004;200(8):951-5. 40. Xiong S, Sharkey AM, Kennedy PR, Gardner L, Farrell LE, Chazara O, et al. Maternal uterine NK cell-activating receptor KIR2DS1 enhances placentation. J Clin Invest. 2013;123(10):4264-72.

41. De Oliveira LG, Lash GE, Murray-Dunning C, Bulmer JN, Innes BA, Searle RF, et al. Role of interleukin 8 in uterine natural killer cell regulation of extravillous trophoblast cell invasion. Placenta. 2010;31(7):595-601. 
534 42. Hamilton S, Oomomian Y, Stephen G, Shynlova O, Tower CL, Garrod A, et al. Macrophages

535 infiltrate the human and rat decidua during term and preterm labor: evidence that decidual

536 inflammation precedes labor. Biol Reprod. 2012;86(2):39.

537 43. Rinaldi SF, Makieva S, Saunders PT, Rossi AG, Norman JE. Immune cell and transcriptomic

538 analysis of the human decidua in term and preterm parturition. Mol Hum Reprod. 2017;23(10):708-

53924.

540 44. Sindram-Trujillo A, Scherjon S, Kanhai H, Roelen D, Claas F. Increased T-cell activation in

541 decidua parietalis compared to decidua basalis in uncomplicated human term pregnancy. Am J

542 Reprod Immunol. 2003;49(5):261-8.

543 45. Pace D, Morrison L, Bulmer JN. Proliferative activity in endometrial stromal granulocytes

544 throughout menstrual cycle and early pregnancy. J Clin Pathol. 1989;42(1):35-9.

545 46. Strunz B, Bister J, Jönsson H, Filipovic I, Crona-Guterstam Y, Kvedaraite E, et al.

546 Continuous human uterine NK cell differentiation in response to endometrial regeneration and

547 pregnancy. Sci Immunol. 2021;6(56).

548 47. Wang F, Jia W, Fan M, Shao X, Li Z, Liu Y, et al. Single-cell Immune Landscape of Human

549 Recurrent Miscarriage. Genomics Proteomics Bioinformatics. 2021.

550 48. Tuckerman E, Mariee N, Prakash A, Li TC, Laird S. Uterine natural killer cells in peri-

551 implantation endometrium from women with repeated implantation failure after IVF. J Reprod

552 Immunol. 2010;87(1-2):60-6.

553 49. Fraser R, Whitley GS, Thilaganathan B, Cartwright JE. Decidual natural killer cells regulate

554 vessel stability: implications for impaired spiral artery remodelling. J Reprod Immunol. 2015;110:54-

55560. 
(A)

(B) Non pregnant 1st trimester 2 2nd trimester 3 3rd trimester
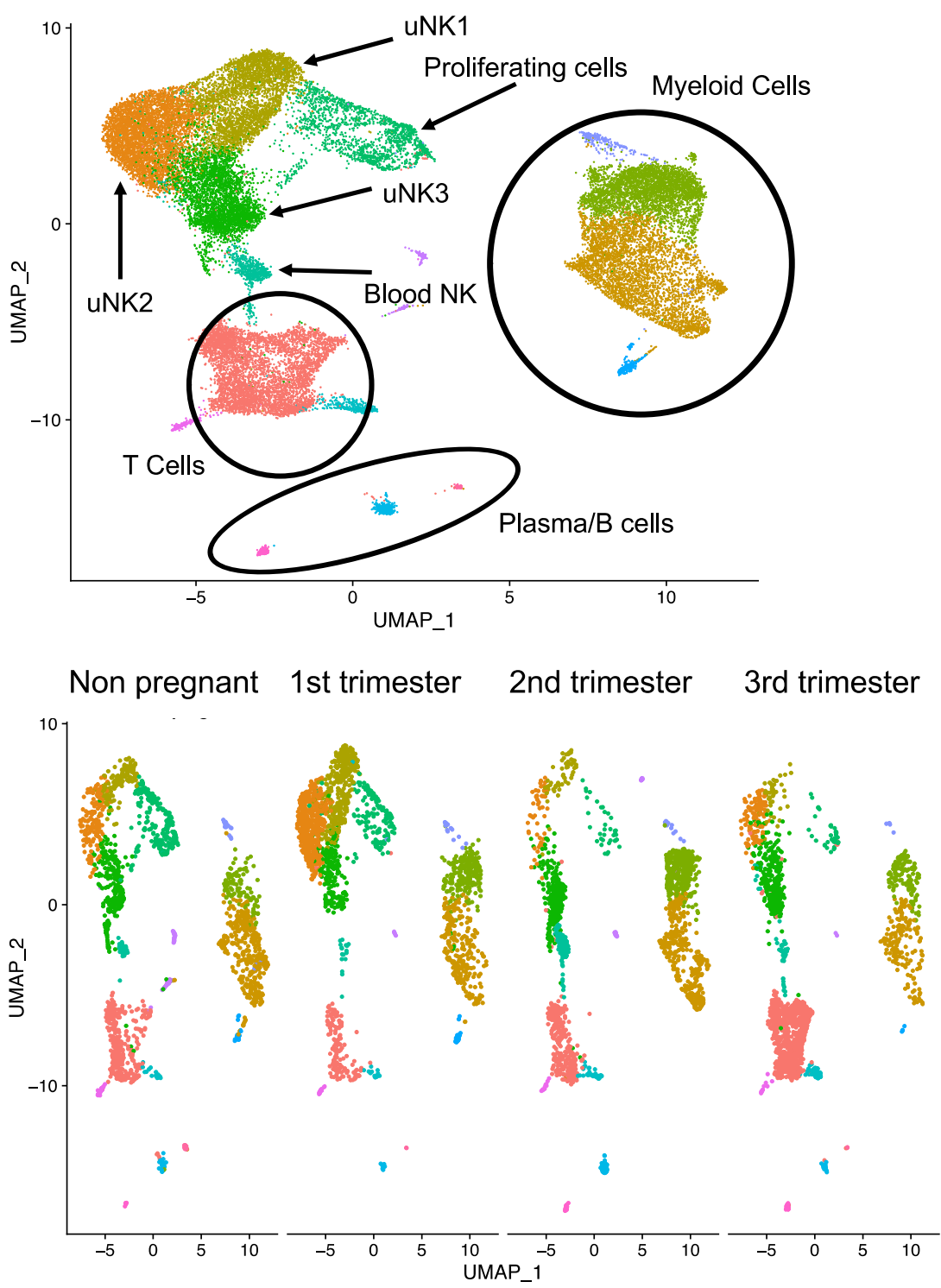

(C)
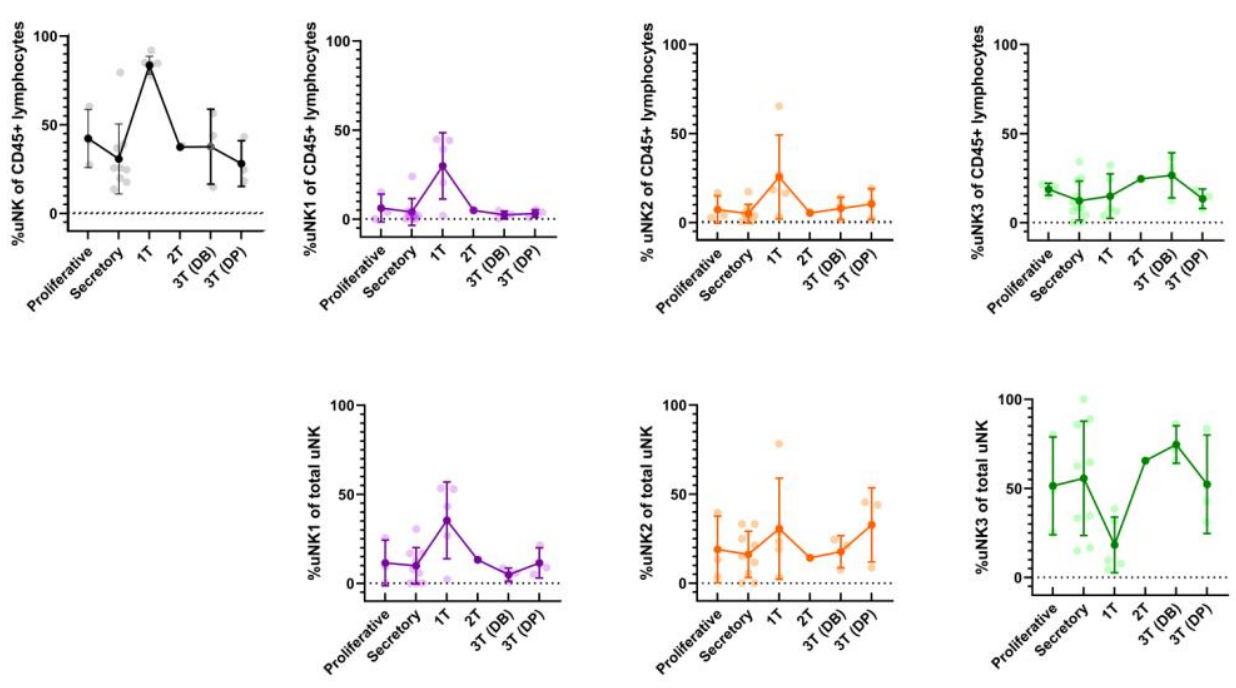
Figure $1-u N K 1,-2$ and -3 are present throughout the human reproductive cycle, by scRNAseq

559 (A) Integrated immune cells from non-pregnant endometrium, and first, second and third trimester decidua, visualised by

560 UMAP. Colours are indicative of clusters and are with appropriate immune cell type. $n=12$ (non-pregnant), $n=5$ (1T),

$561 \mathrm{n}=1(2 \mathrm{~T}), \mathrm{n}=3(3 \mathrm{~T})$. uNK, uterine Natural Killer (B) Immune cells from each of the four stages in the reproductive cycle

562 subset to 2200 cells. Immune cells separated by stage and then visualised by UMAP. Colours are indicative of clusters.

563 (C) Using the scRNA seq dataset, graphs show frequency of total NK from CD45+ lymphocytes and then frequency of

564 each uNK subset (uNK1, -2 -3) both as a proportion of CD45+ lymphocytes and proportion of total uNK cells. Means

565 and standard deviations are shown for $\mathrm{n}=3$ (proliferative), $\mathrm{n}=3$ (secretory) $\mathrm{n}=5(1 \mathrm{~T}), \mathrm{n}=1(2 \mathrm{~T}), \mathrm{n}=3(3 \mathrm{~T}$ DB), $\mathrm{n}=3(3 \mathrm{~T}$

566 DP) DB, decidua basalis; DP, decidua parietalis; 1T, first trimester; 2T, second trimester; 3T, third trimester. 
A
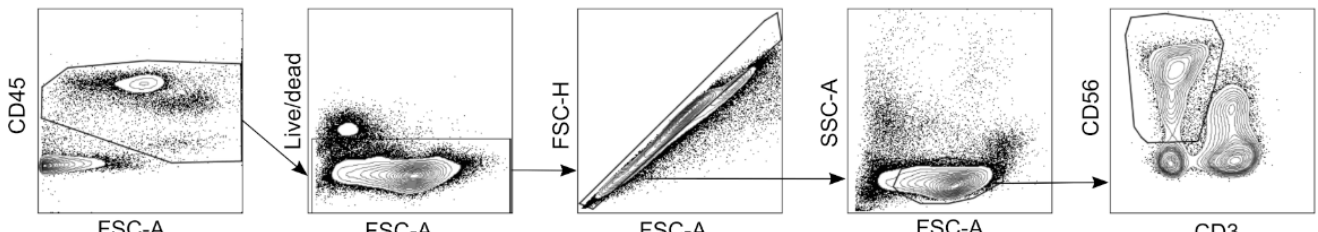

Uterus

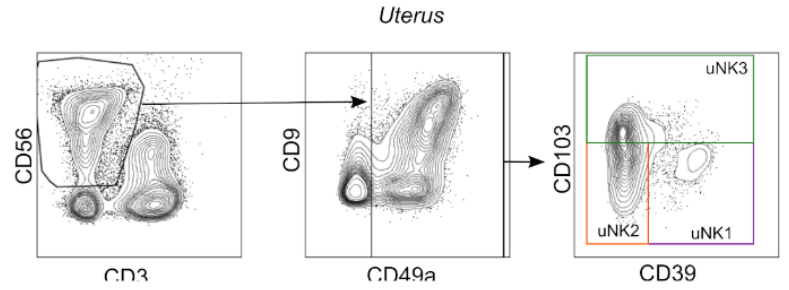

Blood

B

C

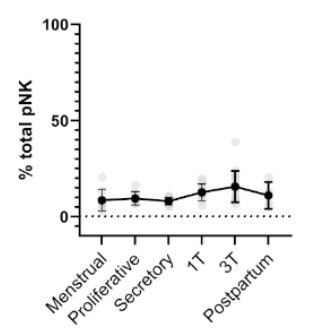

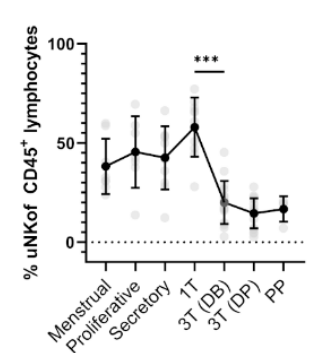
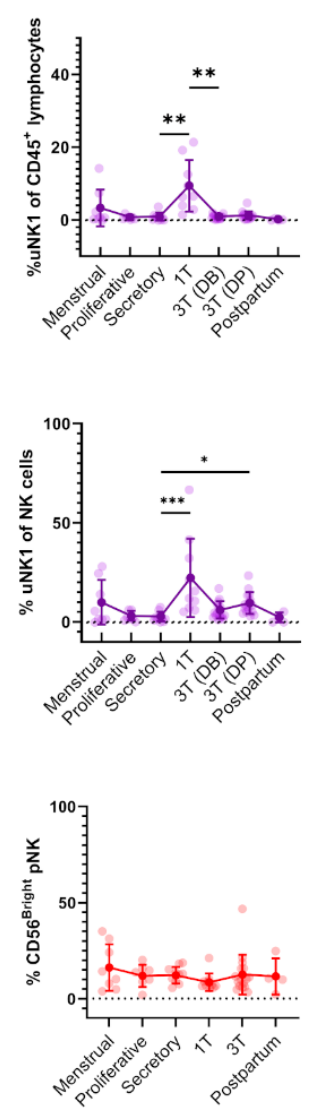
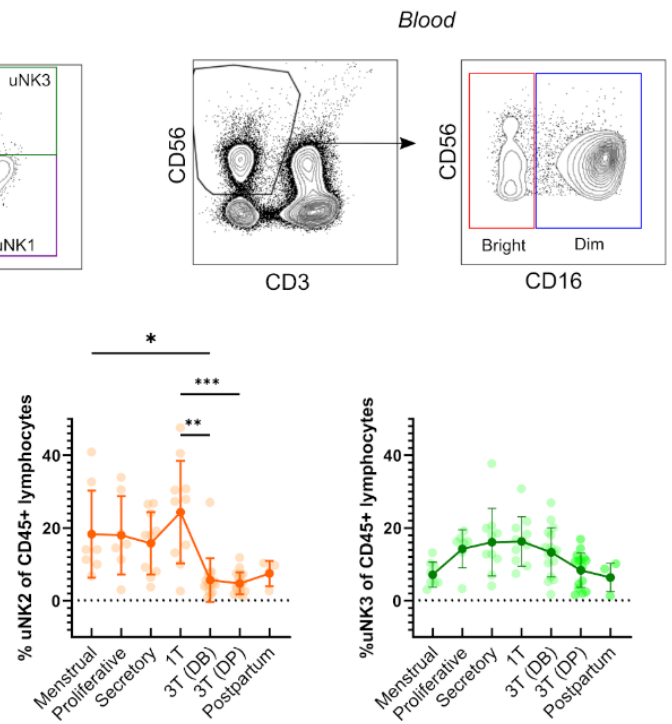

568

569

570

571

572

573

574

575

576

577

578
Figure $2-u N K 1,-2$ and -3 are present throughout the human reproductive cycle, by flow cytometry

(A) FACs gating strategy used to identify three uNK subsets and pNK (representative example shown). Coloured boxes in final plot indicate colour used for that subset in subsequent graphs. (B) Using flow cytometry data, graphs show frequency of total NK from CD45+ lymphocytes and then frequency of each uNK subset (uNK1, -2 -3) both as a proportion of CD45+ lymphocytes and proportion of total uNK cells. Means and standard deviations are shown for $\mathrm{n}=8$ (menstrual), $\mathrm{n}=7$ (proliferative), $\mathrm{n}=10$ (secretory) $\mathrm{n}=10(1 \mathrm{~T}), \mathrm{n}=16$ (3T DB), $\mathrm{n}=16(3 \mathrm{~T} \mathrm{DP}), \mathrm{n}=4$ (postpartum). Statistical testing was done using Kruskal Wallis with a post-hoc Dunn test $* \mathrm{p}<0.05, * * \mathrm{p}<0.01, * * * \mathrm{p}<0.001 * * * *$ $\mathrm{p}<0.0001$ (C) Using flow cytometry data, graphs show frequency of total NK from CD45+ lymphocytes and then frequency of each pNK subset (CD56Brightand CD56Dim) as a proportion of total pNK. n numbers for each group are the same as B. DB, decidua basalis; DP, decidua parietalis; 1T, first trimester; 2T, second trimester; 3T, third trimester. 
A

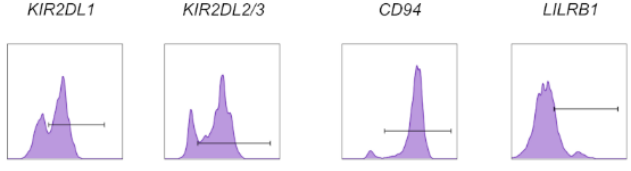

B
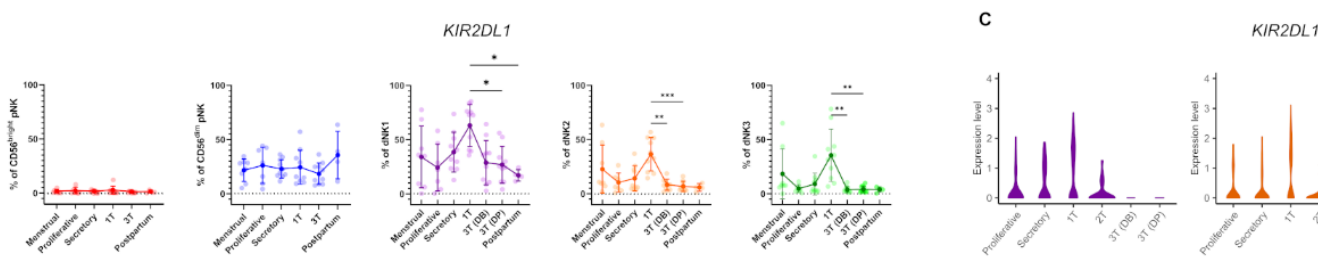

KIR2DL2/3
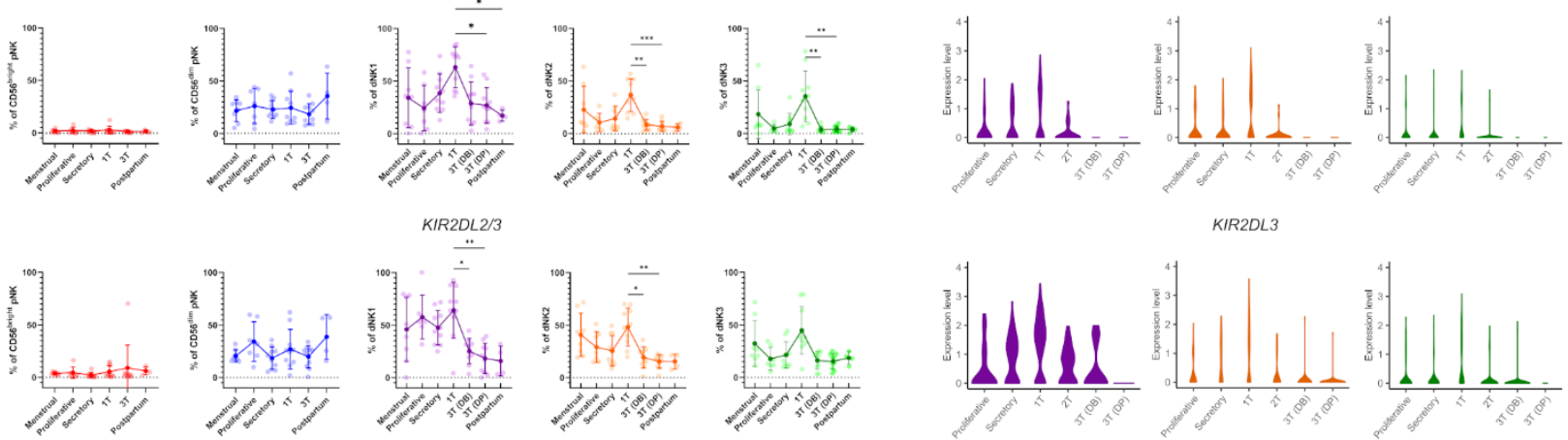

KIR2DL3
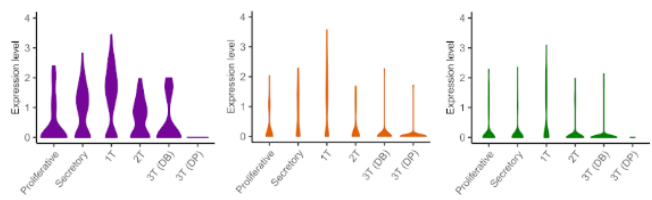

LILRB1
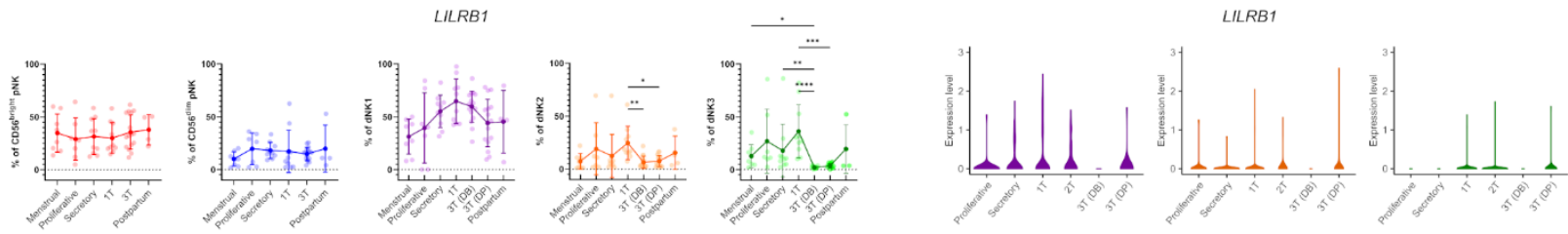

CD94
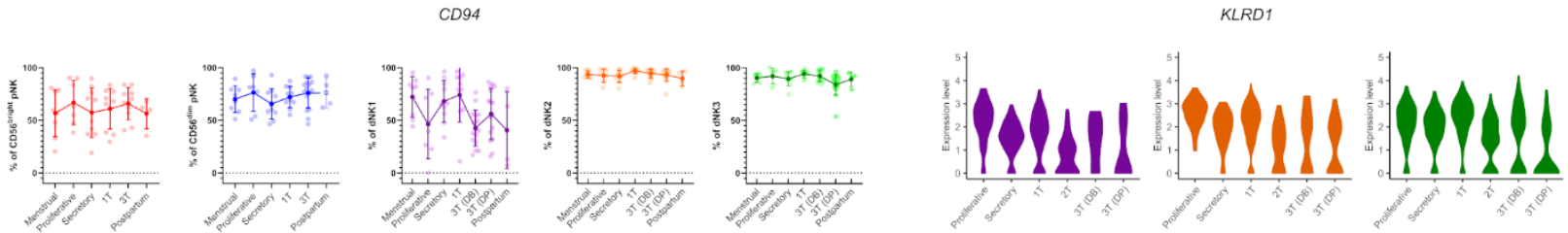

580

581

Figure $3-u N K$ upregulate expression of KIR and LILRB1 in first trimester

583

(A) Uterine and peripheral NK cells taken at different stages of the reproductive cycle were freshly stained for phenotypic markers. Representative staining from secretory phase uNK1 is shown. The positive gates were set by reference to FMO controls. (B) Graphs showing frequencies of KIR2DL1, KIR2DL2/3, LILRB1 and CD94 on pNK and uNK subsets.

Means and standard deviations are shown for $\mathrm{n}=8$ (menstrual), $\mathrm{n}=7$ (proliferative), $\mathrm{n}=10$ (secretory) $\mathrm{n}=10(1 \mathrm{~T}), \mathrm{n}=16$ (3T DB), $n=16$ (3T DP), $n=4$ (postpartum). Statistical testing was done using Kruskal Wallis with a post-hoc Dunn test $* \mathrm{p}<0.05$, ** $\mathrm{p}<0.01, * * * \mathrm{p}<0.001$. (C) Violin plots showing corresponding mRNA expression in uNK subsets over the reproductive cycle as determined by scRNAseq. DB, decidua basalis; DP, decidua parietalis; 1T, first trimester; $2 \mathrm{~T}$,

590 second trimester; 3T, third trimester. 
A

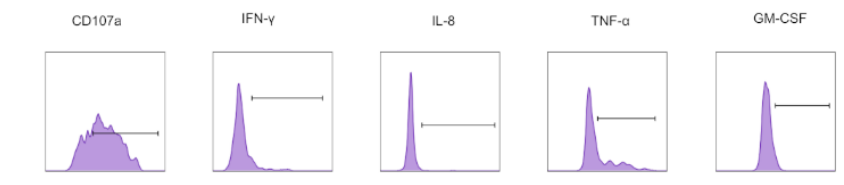

B

CD107a
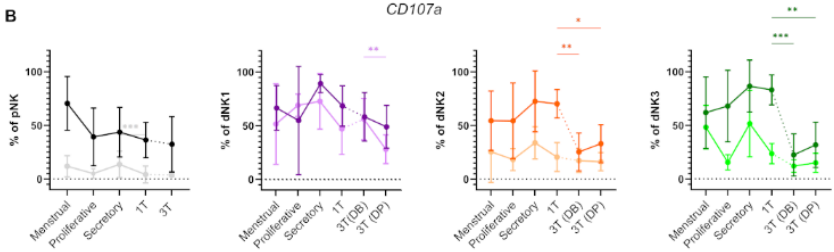

IFN-Y
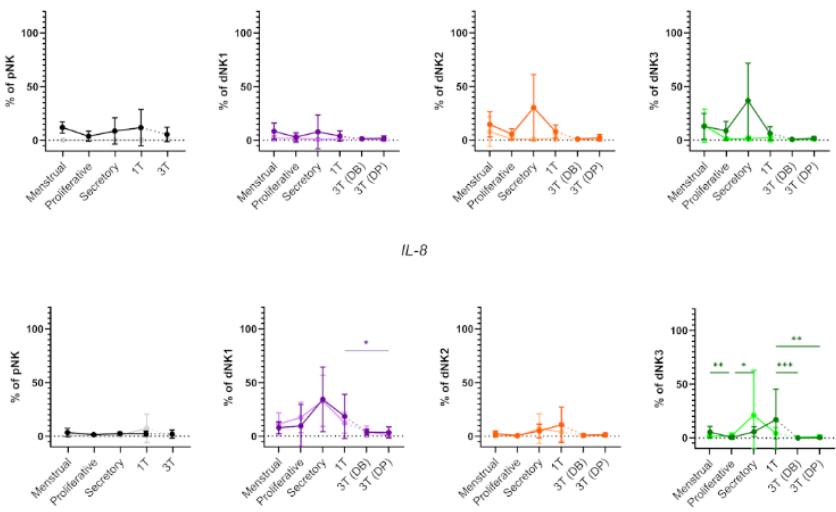

TNF- $Q$
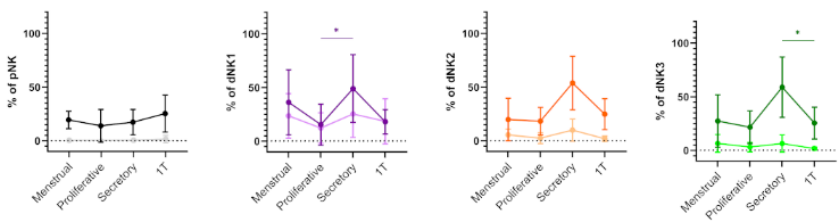

GM-CSF
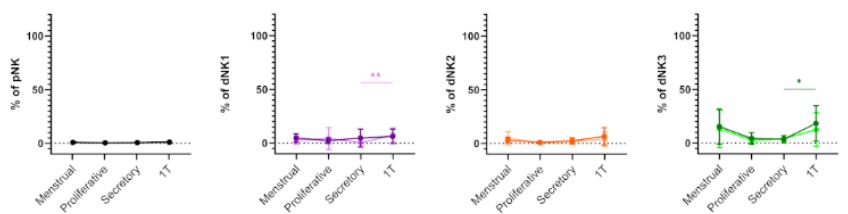
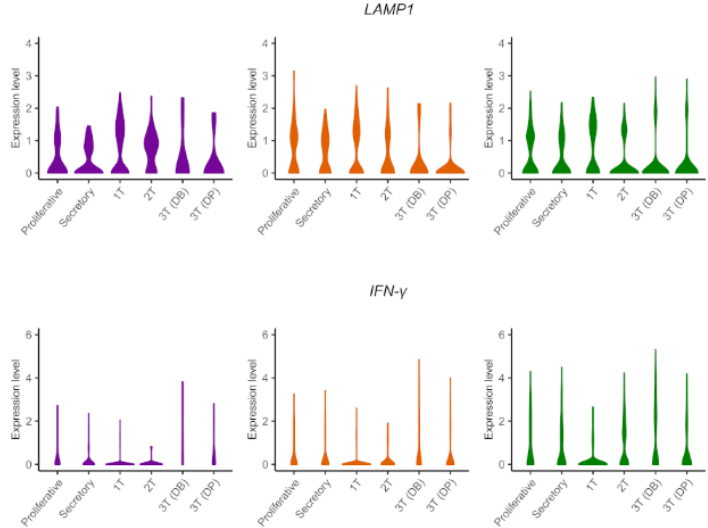

CXCL-8
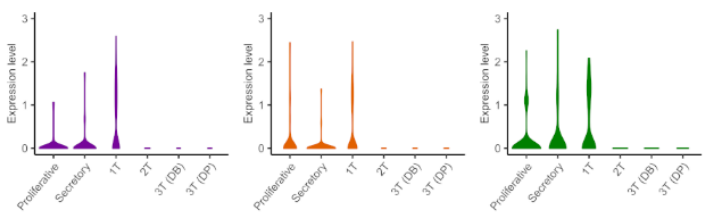

$T N F-Q$
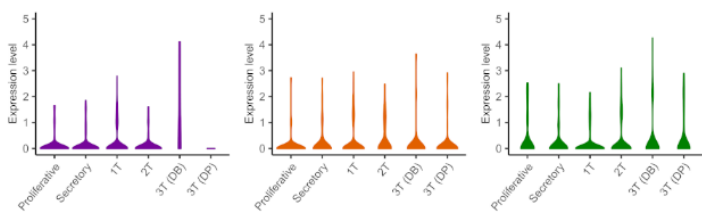

CSF-2
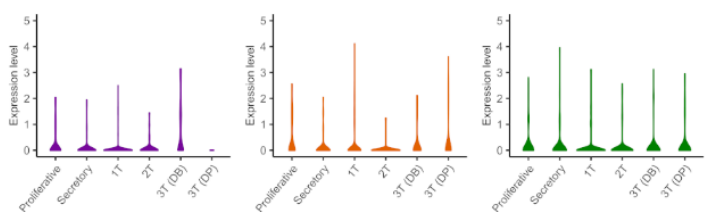

Figure 4 uNK are most active at the time of implantation.

(A) Uterine and peripheral NK cells taken at different stages of the reproductive cycle were cultured with or without

PMA and ionomycin stimulation and assessed for degranulation (CD107a) and production of IFN $\gamma$, IL-8, TNF $\alpha$ and GM-

CSF. Representative staining from secretory phase uNK1 is shown. The positive gates were set by reference to FMO shown for $\mathrm{n}=5$ (menstrual), $\mathrm{n}=6$ (proliferative), $\mathrm{n}=10$ (secretory) $\mathrm{n}=10(1 \mathrm{~T}), \mathrm{n}=16$ (3T DB), $\mathrm{n}=16$ (3T DP). 

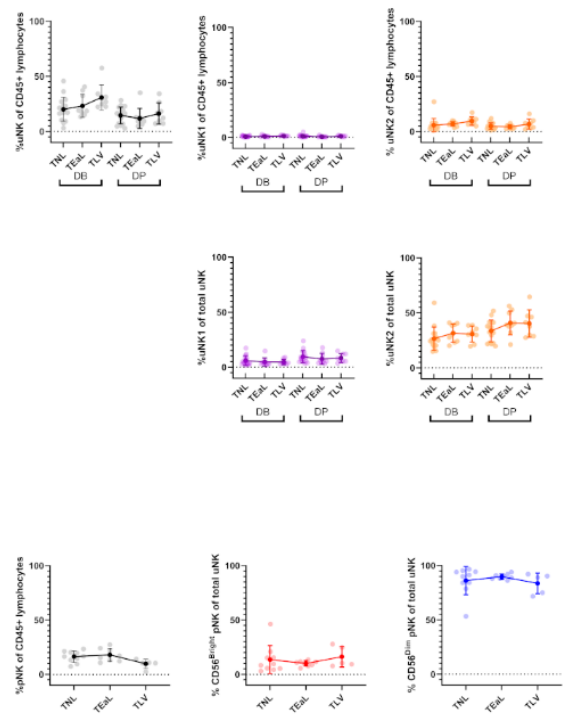
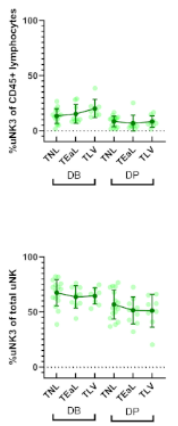
$\stackrel{D B}{D}$

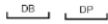
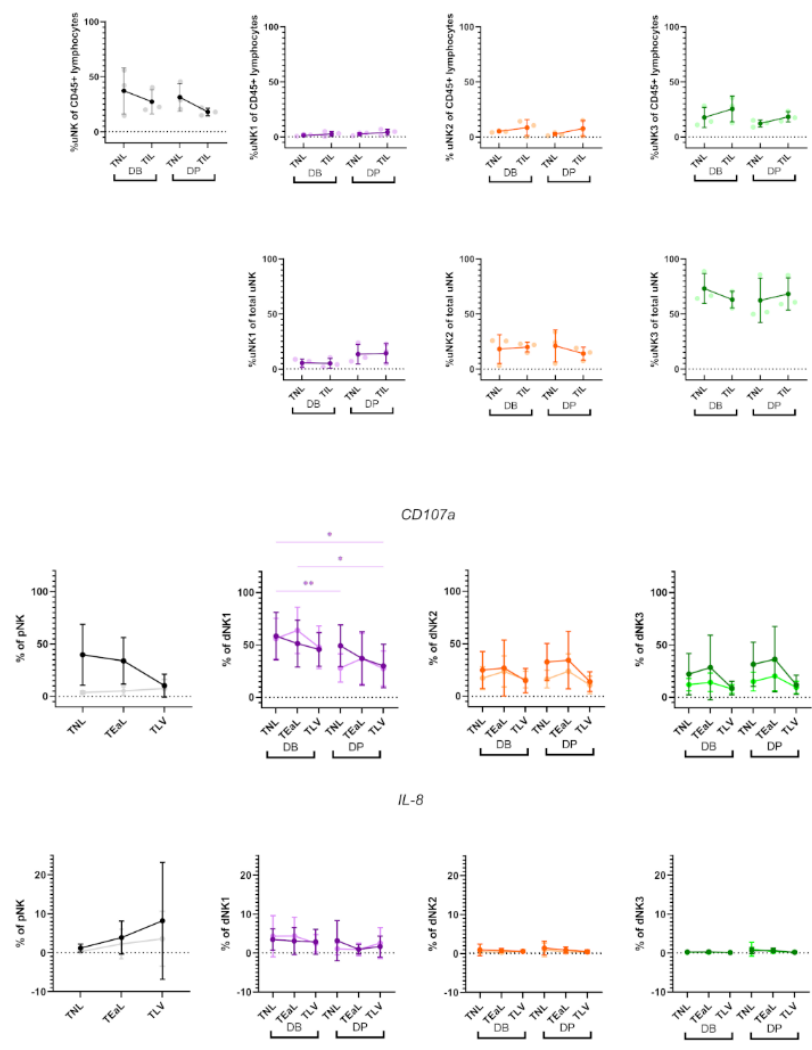

606

607

608

609

610

611

612

613

614

615

616

617

618

619

620

621

622

623

624

Figure 5 - uNK phenotype and function do not change in labour

(A) The FACs gating strategy shown in Figure 2A was used to identify the three uNK subsets. Graphs show frequency of total NK from CD45+ lymphocytes and then frequency of each uNK subset (uNK1, -2 -3) both as a proportion of CD45+ lymphocytes and proportion of total uNK cells. Graphs are divided to show results from both decidua basalis and decidua parietalis. Means and standard deviations are shown for $n=16$ (TNL), $n=9$ (TEaL), $n=9$ (TLV). Statistical testing was done using Kruskal Wallis with a post-hoc Dunn test $* \mathrm{p}<0.05$. (B) Using the scRNA seq dataset, graphs show frequency of total NK from CD45+ lymphocytes and then frequency of each uNK subset (uNK1, -2 -3) both as a proportion of CD45+ lymphocytes and proportion of total UNK cells. Graphs are divided to show results from both decidua basalis and decidua parietalis. Means and standard deviations are shown for $\mathrm{n}=3$ (TNL), $\mathrm{n}=3$ (TIL). (C) The FACs gating strategy shown in Figure $2 \mathrm{C}$ was used to identify the two pNK subsets. Using flow cytometry data, graphs show frequency of total NK from CD45+ lymphocytes and then frequency of each pNK subset (CD56Bright and CD56Dim) from total NK. $n$ numbers for each group are the same as (A). (D) Uterine and peripheral NK cells were cultured as described in Figure 4A. Graphs showing frequencies of CD107a and IL-8 on pNK and uNK subsets. Unstimulated cells are represented by lighter lines and stimulated cells by darker lines. $\mathrm{n}$ numbers for each group are the same as (A). Statistical testing was done using Kruskal Wallis with a post-hoc Dunn test $* \mathrm{p}<0.05$. DB, decidua basalis; DP, decidua parietalis; TNL, term non-labouring; TEaL, term early labouring; TLV, term vaginal birth; TIL, term in labour 